\title{
Redes de sensores IoT em edifícios inteligentes: uma avaliação de desempenho usando modelos de filas
}

\author{
Brena Santos, Francisco Airton Silva e André Soares \\ ${ }^{1}$ Universidade Federal do Piauí (UFPI) \\ Teresina - PI - Brasil \\ E-mail: \{brenamaia, faps, andre.soares\}@ufpi.edu.br
}

\begin{abstract}
Buildings in large cities are becoming more and more intelligent with the adoption of IoT devices. The IoT sensors and actuators must be efficient as malfunctions can cause major damage to property and perhaps loss of life. To assess the performance of building monitoring systems, some studies have used analytical models. However, some points have not yet been explored in the literature, such as computational capacity analysis, representation of the number of cores per machine and sensors grouped by location. This work proposes a queuing model to evaluate the performance of a smart building infrastructure with multiple processing support.
\end{abstract}

Resumo. Os edifícios das grandes cidades estão cada vez mais inteligentes com a adoção de dispositivos de IoT. Os sensores e atuadores IoT devem ser eficientes pois o mau funcionamento pode causar danos patrimoniais e talvez perda de vidas. Para avaliar o desempenho de sistemas de monitoramento de edifícios, alguns estudos usaram modelos analíticos. No entanto, alguns pontos ainda não foram explorados na literatura, tais como a análise da capacidade de recursos computacionais adequados à demanda de dados, representação do número de núcleos por máquina e o agrupamento de sensores por localização. Este trabalho propõe um modelo de filas para avaliar o desempenho de uma infraestrutura de edifício inteligente com suporte de múltiplas camadas de processamento.

\section{Introdução}

A Internet das Coisas (Internet of Things - IoT) se destina à conectividade onipresente entre diferentes entidades ou "coisas" [Mena et al. 2018]. A IoT é promissora devido à sua importância em muitas aplicações de comércio, indústria e educação [Said and Masud 2013]. Dispositivos IoT podem ser usados, por exemplo, para automatizar atividades em casas inteligentes [Ajao et al. 2020], gerenciar tráfego a partir de redes veiculares [Wu et al. 2020], e monitoramento de saúde [Sokullu et al. 2020]. A capacidade de identificar comportamentos anormais em um ambiente monitorado usando IoT tem sido bastante útil em casas e edifícios inteligentes.

Originalmente, a tecnologia de casa inteligente era usada para controlar sistemas ambientais como iluminação e aquecimento. No entanto, há algum tempo, o uso da IoT permite que quase todos os componentes elétricos da casa possam ser conectados a um sistema. Uma casa inteligente pode fornecer serviços baseados em contexto para proprietários de residências usando sensores e outras tecnologias. A partir do conceito de casas inteligentes pode-se estender o uso da IoT para um contexto mais amplo: os edifícios inteligentes. Os edifícios por questões de grandeza e complexidade exigem infraestruturas 
computacionais mais sofisticadas e um preparo para atender grande demanda de geração de dados.

Como uma estratégia para mitigar essa demanda de dados, a computação de borda surgiu para otimizar o processamento mais próximo da fonte dos dados [Xu et al. 2020]. No entanto, para sistemas com muitos parâmetros envolvidos, avaliações com experimentos reais podem ser custosos por existirem muitas possibilidades de configurações. Modelos analíticos podem ser úteis nesse contexto, permitindo previsões com base em probabilidades.

A teoria das filas é um método matemático para analisar diferentes tipos de sistemas e observar seu comportamento em relação ao desempenho do sistema e a qualidade do serviço. Os modelos de filas são simples e didáticos, porém muito poderosos [Attia et al. 2019, Memon et al. 2019, Arbib et al. 2019]. As aplicações da teoria de filas geralmente possuem dois objetivos: prever o desempenho do sistema e encontrar um design de sistema para melhorar o desempenho na fase de planejamento [Jafarnejad Ghomi et al. 2019].

Existem alguns trabalhos que desenvolveram modelos analíticos para avaliar os recursos dos sistemas IoT em casas e edifícios inteligentes. Alguns deles focaram em garantir apenas a eficácia do sistema [Nabih et al. 2011, Novák et al. 2012, Fanti et al. 2014b, Garcia et al. 2018]. Outros analisaram a eficiência energética [Fanti et al. 2014a, Fanti et al. 2018, Wang et al. 2013]. Em [Ajao et al. 2020] o sistema foi analisado utilizando as métricas tempo de resposta e porcentagem de erro. [Oueida et al. 2018] observa o tempo total de atendimento da requisição, a taxa de utilização de recursos e o tempo médio de espera. Apenas [Oueida et al. 2018] explorou camadas de computação, integrando seu modelo com nuvem e computação de borda. No entanto, [Oueida et al. 2018] não explora performance de componentes computacionais e não oferece as mesmas contribuições que a arquitetura deste trabalho. Além disso, somente este trabalho considerou sensores agrupados por localização, característica essencial quando se faz monitoramento de mais de um ambiente.

Portanto, o presente artigo propõe um modelo de filas para avaliar sistemas IoT para edifícios inteligentes suportados por borda-névoa. As contribuições deste trabalho são as seguintes:

- Um modelo de filas, que é uma ferramenta útil para projetistas de sistemas avaliarem o desempenho de arquiteturas para edifícios inteligentes suportados por recursos de borda-névoa, antes mesmo de sua implementação. O modelo permite configurar diversos parâmetros de acordo com a necessidade do projetista, incluindo quantidade de nós, tempos de serviços, tamanho limite de filas, dentre outros.

- Um conjunto de análises numéricas que serve como um guia para analisar o desempenho de um edifício inteligente. O cenário monitorado analisa o comportamento do sistema ao alterar a quantidade de nós da névoa. Consideramos métricas como tempo médio de resposta (Mean Response Time - MRT), utilização dos recursos, taxa de descarte e taxa de transferência.

O restante deste trabalho está organizado da seguinte forma: A Seção 2 apresenta os trabalhos relacionados, comparando-os com nossa proposta. A Seção 3 descreve a arquitetura que foi utilizada como base para a construção do modelo. Na Seção 4 apre- 
sentamos o modelo de filas proposto. A Seção 5 apresenta os resultados obtidos nas análises. Por fim, na Seção 6 encontra-se a conclusão e trabalhos futuros.

\section{Trabalhos Relacionados}

Oito estudos relacionados foram selecionados e ordenados por ano, o mais antigo é de 2011. Os trabalhos foram coletados a partir de buscas no Google Scholar. As strings de busca continham palavras referentes a modelos analíticos (ex: Petri Nets, Markov Chain e Queue) e casas ou edifícios inteligentes. A Tabela 1 apresenta uma comparação dos trabalhos coletados, destacando as principais diferenças de nossa proposta. Em seguida, os trabalhos são discutidos de forma agrupada de acordo com cada critério de comparação.

Tabela 1. Trabalhos Relacionados

\begin{tabular}{|c|c|c|c|c|c|}
\hline Trabalho Relacionado & Contexto de Aplicação & Métricas & $\begin{array}{l}\text { Análise da } \\
\text { Capacidade } \\
\text { de Recursos }\end{array}$ & $\begin{array}{l}\text { Sensores } \\
\text { Agrupados por } \\
\text { Localização }\end{array}$ & $\begin{array}{l}\text { Representa } \\
\text { Número de } \\
\text { Núcleos por } \\
\text { Máquina }\end{array}$ \\
\hline [Nabih et al. 2011] & Casa inteligente & Eficácia do sistema & Não & Não & Não \\
\hline [Novák et al. 2012] & Casa inteligente & Eficácia do sistema & Não & Não & Não \\
\hline [Wang et al. 2013] & Edifício inteligente & Eficiência Energética & Não & Não & Não \\
\hline [Fanti et al. 2014a] & Edifício inteligente & Eficiência energética & Não & Não & Não \\
\hline [Fanti et al. 2014b] & Casa inteligente & $\begin{array}{l}\text { Eficácia do sistema, } \\
\text { vivacidade, limitação } \\
\text { e reversibilidade }\end{array}$ & Não & Não & Não \\
\hline [Fanti et al. 2018] & Edifício inteligente & Eficiência energética & Não & Não & Não \\
\hline [Garcia et al. 2018] & Casa Inteligente & Eficácia do sistema & Não & Não & Não \\
\hline [Ajao et al. 2020] & Casa inteligente & $\begin{array}{l}\text { Porcentagem de erro, } \\
\text { Tempo de resposta. }\end{array}$ & Não & Não & Não \\
\hline Este trabalho & Edifício inteligente & $\begin{array}{l}\text { Utilização de re- } \\
\text { cursos, MRT, Taxa } \\
\text { de descarte, Taxa } \\
\text { de transferência, } \\
\text { Número de trabalhos } \\
\text { no sistema. }\end{array}$ & Sim & Sim & Sim \\
\hline
\end{tabular}

Componentes principais da arquitetura - Alguns trabalhos fazem o uso de sensores para buscar melhorias na qualidade de vida de idosos, monitorando-os de forma não invasiva para detectar inatividades e possíveis acidentes domésticos. [Garcia et al. 2018], [Fanti et al. 2014b] e [Novák et al. 2012] utilizam os dados coletados para avaliar a necessidade de emitir notificações para pessoas que estão fora do ambiente observado. Ambos fizeram uso de algum modelo analítico (rede de Petri ou modelo de Markov) e os avaliam considerando cenários diversos, verificando a eficácia do sistema. [Garcia et al. 2018] verificam, a partir de sensores térmicos e de contato, atividades realizadas em uma cozinha com o objetivo de monitorar o comportamento de idosos. [Fanti et al. 2014b] buscam monitorar a vida do habitante e detectar inatividades e acidentes, além de avaliar a gravidade do acidente para comunicar familiares e serviços de urgência. [Novák et al. 2012] apresentam um método para detecção de anomalias nas atividades do usuário, utilizando 
sensores discretos que classificam a presença do usuário em suas atividades habituais. Apesar dos trabalhos citados abordarem o possível envio de mensagens para ambientes externos, os autores não se atentam ao modo como a comunicação será feita, como também não focam no processamento remoto. Nossa proposta explora não apenas a IoT, mas também as camadas de borda e névoa como recursos complementares.

Métricas - As métricas de desempenho ajudam a entender como o sistema se comporta em diferentes cenários de uso. [Nabih et al. 2011, Novák et al. 2012, Fanti et al. 2014b] utilizam modelos para monitorar atividades em casas inteligentes. Eles utilizaram como métrica a eficácia do sistema, ou seja, definem cenários e verificam se o sistema reage da maneira esperada de acordo com as ações detectadas. Em [Novák et al. 2012], os autores utilizaram um conjunto de dados contendo informações sobre transições de idosos entre diferentes cômodos de um apartamento. Comportamentos fora do esperado foram adicionados artificialmente ao sistema como meio de teste. Em [Fanti et al. 2014b] os autores realizam uma simulação que permite verificar se os protocolos de emergência são executados corretamente e se as propriedades de vivacidade, limitação e reversibilidade são preservadas. Os autores em [Fanti et al. 2014a, Fanti et al. 2018, Wang et al. 2013] analisam dispositivos de casas inteligentes, a partir da análise de eficiência energética pode ser solicitada a redução do consumo de energia dos dispositivos do edifício. Os autores em [Ajao et al. 2020] projetaram uma sala inteligente com controle de janela automatizado, que pode abrir e fechar automaticamente com base nas mudanças nas condições climáticas. Para a avaliação do sistema, foram observadas as métricas tempo de resposta e porcentagem de erro. Algumas métricas analisadas em trabalhos citados também foram utilizadas neste trabalho. No entanto, apenas o nosso trabalho analisou as seguintes métricas: MRT, taxa de descarte, taxa de transferência, utilização de recursos computacionais e número de trabalhos no sistema. Essas métricas são importantes para analisar se o sistema possui funcionamento adequado. Ademais, este trabalho não se limita a avaliar sensores ou condições ambientais específicas. O modelo proposto é parametrizável para qualquer tipo de dado coletado por conjunto de sensores.

Três parâmetros usados para a comparação dos trabalhos aumentam significativamente a contribuição deste estudo, pois são características encontradas apenas neste trabalho. Análise da capacidade de recursos refere-se à avaliação de como o sistema se comporta de acordo com a quantidade de recursos disponíveis. Essa análise permite prever se o sistema irá conseguir atender as solicitações de maneira satisfatória, podendo também evitar o desperdício de recursos computacionais. Em nosso trabalho, analisamos o comportamento do sistema ao alterar a quantidade de nós na névoa. Sensores agrupados por localização se refere ao modo como o modelo representa diferentes conjuntos de sensores. Nosso modelo permite a atribuição de diferentes taxas de chegada, a depender de sua localização. Em nosso modelo, esses locais podem ser vistos como salas de um edifício. Esta funcionalidade visa tornar o modelo mais realista, pois dependendo da localização, a geração de dados pode ser diferente, exigindo envio de dados. Existem diferenças deste trabalho em relação aos trabalhos relacionados com frequências distintas. Nosso modelo também é o único que representa o número de núcleos de processamento por máquina. A camada de névoa possui máquinas com múltiplos núcleos. O modelo permite variar a capacidade da névoa alterando o número de máquinas e a quantidade de núcleos em cada uma delas. Acreditamos que esse recurso é fundamental para representar 
arquiteturas com precisão.

\section{Arquitetura do Sistema}

Esta seção apresenta a arquitetura explorada no trabalho. Por questões de clareza da proposta, três aspectos são discutidos: arquitetura geral do sistema, ciclo de vida das mensagens e suposições/discussões.

Arquitetura geral do sistema - A Figura 1 ilustra a arquitetura de uma infraestrutura para monitoramento de segurança em edifícios inteligentes. Os sensores podem ser de diversas naturezas e ter objetivos distintos, porém como forma de ilustração damos como exemplos alguns possíveis tipos de sensores. O edifício pode detectar movimentação inesperada nos ambientes (por exemplo, em horários em que não é permitido o acesso a determinados locais) e notificar aos seguranças. Além disso, pode detectar sinais de incêndio e notificar aos bombeiros. A arquitetura é composta por duas camadas de computação: (i) camada de computação de borda em salas do edifício, para a integração de dados de sensores de segurança; (ii) camada de computação em data centers de névoa, para o acesso de clientes internos (por exemplo, bombeiros e policiais). A camada de computação na borda permite o monitoramento e agregação de dados sobre a segurança em tempo real, usando detectores de fumaça e de movimento para coletar e processar periodicamente dados sobre o ambiente de cada sala. Para fornecer os serviços de monitoramento de segurança, a camada de computação na borda é projetada com vários nós para realizar a coleta e o processamento de dados em todas as salas de todos os andares do edifício. A camada de computação na névoa é composta por: um gateway de névoa-borda para agrupamento de dados e balanceamento de carga entre as duas camadas; um conjunto de nós de névoa para processamento paralelo de dados; e uma estação onde os dados são disponibilizados para o acesso de clientes internos.

Ciclo de vida das mensagens - A arquitetura indica também o ciclo de vida dos pacotes de dados e comportamentos operacionais do sistema de monitoramento de segurança. Os dados são coletados periodicamente pelos detectores de movimento, de fumaça e de aquecimento, em seguida são agrupados e encapsulados por dispositivos de borda como um alerta de segurança. Esses alertas são então transmitidos para a névoa através de um gateway. O gateway Borda-Névoa desempenha um papel como porta de entrada para distribuição de dados e balanceamento de carga para os nós da névoa. As mensagens são processadas em nós da névoa por aplicativos especializados, que são personalizados para o tipo de alerta. Os dados processados na camada de névoa são entregues diretamente aos clientes internos (seguranças e/ou bombeiros). Visto isso, podem surgir questionamentos como: "qual o impacto da variação da taxa de chegada (Arrival Rate - AR) de dados, nas métricas de desempenho dos serviços prestados (por exemplo, taxa de utilização, tempo médio de resposta)?"; e "como uma configuração específica e sua variação impactam nas métricas de desempenho, desde o recebimento dos dados dos sensores até o acesso por parte dos clientes internos?".

Suposições e discussões - Para simplificar a modelagem, algumas suposições sobre a arquitetura e as operações da infraestrutura IoT em consideração são fornecidas a seguir.

- Camada de borda 


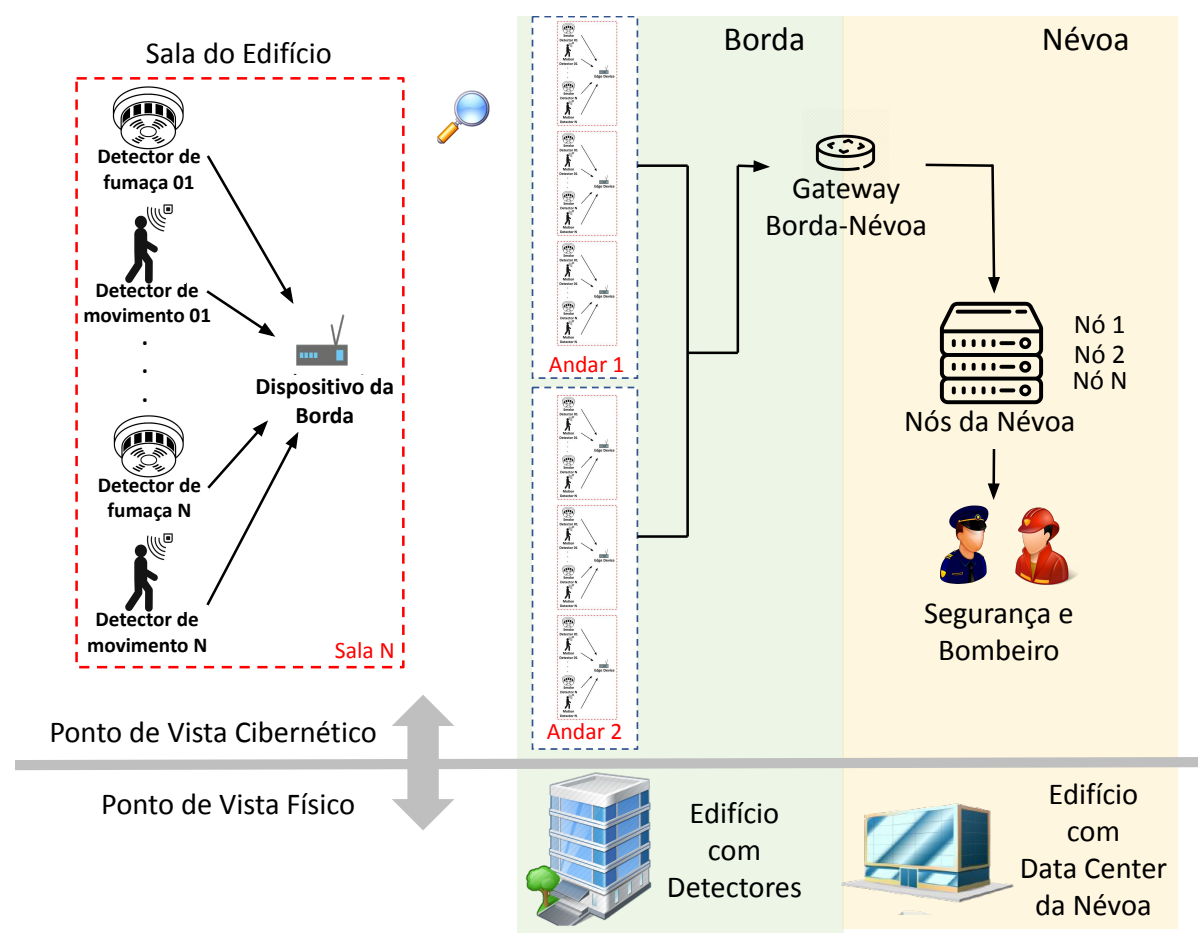

Figura 1. Visão geral de uma arquitetura que permite monitorar vários sensores em um edifício inteligente.

- [b1]: A geração de dados foi modelada para todos os sensores ativos em uma sala, conectados a um dispositivo de borda que também está instalado na sala.

- [b2]: Não levamos em consideração a latência de comunicação entre os sensores e os dispositivos de borda. Na prática, a conexão é formada por comunicação sem fio. No entanto, simplificamos o impacto negativo da comunicação de curta distância na camada de borda nas métricas de desempenho geral.

- [b3]: A latência de comunicação da conexão entre as camadas de borda e névoa é assumida como um atraso na propagação de dados de cada nó de borda para a camada de névoa, que é levado em consideração na modelagem.

- [b4]: A coleta de dados de cada sensor é independente uma da outra, sendo assim, a chegada de solicitações é distribuída exponencialmente com uma taxa de chegada $\lambda$.

- Camada de névoa

- [n1]: Não levamos em consideração um balanceamento de carga sofisticado na camada de névoa. Os trabalhos recebidos no gateway de névoa são igualmente distribuídos para cada um dos nós na camada de névoa. Para simplificar a modelagem, não nos atentamos ao problema de balanceamento de carga de nós de névoa.

- [n2]: Nas análises neste artigo consideramos nós com configurações iguais porém o modelo permite que o avaliador possa configurar como desejar de forma heterogênea.

- Infraestrutura IoT 
- [i1]: O desempenho das transações de dados entre os sensores de IoT e clientes internos (policiais e/ou bombeiros) é o foco principal da modelagem, portanto, o envolvimento de componentes físicos e sua disponibilidade operacional são minimizados. Não consideramos comportamentos de falha e recuperação de componentes na modelagem para avaliação de desempenho.

- [i2]: Nosso foco principal é (i) explorar o gargalo na transmissão de dados de segurança em tempo real, e (ii) explorar o impacto da alteração da configuração da camada de névoa nas métricas de desempenho.

\section{Modelo de Fila}

A Figura 2 ilustra um modelo baseado na teoria das filas para a arquitetura apresentada. O modelo possui vários pontos de entrada e um ponto de saída. O Java Modeling Tools (JMT) foi usado para modelar e avaliar o cenário proposto. JMT é um kit de ferramentas open source para analisar e avaliar o desempenho de sistemas de comunicação baseado na teoria de filas [Fishman 2013]. A Tabela 2 descreve todos os elementos do modelo.

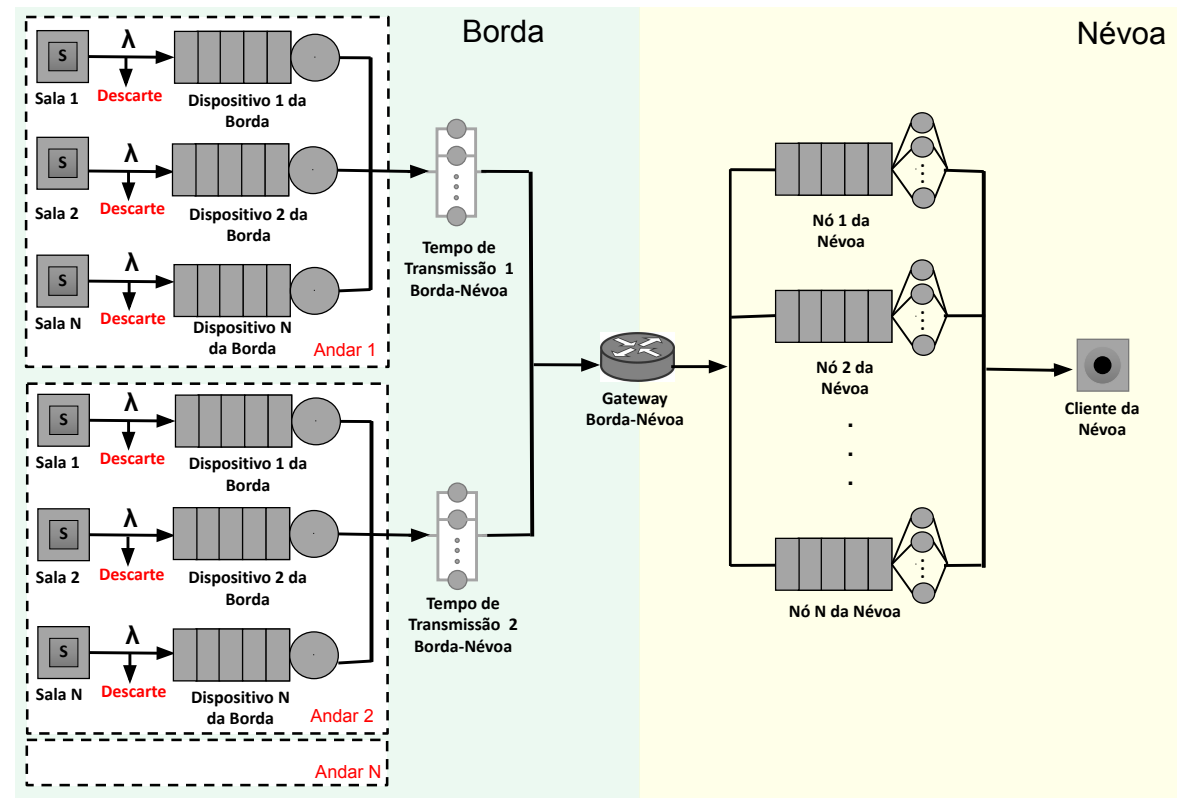

Figura 2. Modelo de filas de uma arquitetura de edifício inteligente composta por múltiplas camadas.

O fluxo de dados no modelo ocorre da esquerda para a direita. As múltiplas entradas do modelo correspondem às salas do edifício. Cada sala possui sensores que geram solicitações dentro de um intervalo de tempo predefinido, e um dispositivo de borda que funciona como uma passagem entre a borda e a camada de névoa. Tal dispositivo de borda é representado por uma fila e um servidor interno exclusivo. As salas possuem $n$ sensores, quantidade que pode variar de acordo com as dimensões da sala, já que ambientes muito grandes podem precisar de vários sensores para cobrir toda a sua área. A taxa de chegada dependerá da quantidade de sensores e da distribuição da geração de dados. Quando a taxa de chegada de dados é maior do que o sistema pode suportar, os dados são descartados. Consideramos que as salas estão organizadas em andares. Cada andar 
Tabela 2. Descrição dos componentes do modelo

\begin{tabular}{lll}
\hline Tipo & Elemento & Descrição \\
\hline & Sensores & Responsável pela geração dos dados. \\
& Dispositivo da Borda & Responsável pelo pré-processamento dos dados. \\
Processamento & Gateway Borda-Névoa & $\begin{array}{l}\text { Responsável pelo encaminhamento dos dados da borda para a névoa. } \\
\text { Salva os dados, processa e encaminha informações e/ou alertas para } \\
\end{array}$ \\
& Nó da Névoa & policiais e/ou bombeiros. \\
\hline Comunicação & Tempo de Propagação Borda- & Atraso na propagação dos dados da borda. \\
\hline Estado & Névoa & Representa o fim do processamento dos dados e disponibilidade para \\
Execução & Cliente da Névoa & policiais e bombeiros. \\
\hline
\end{tabular}

tem uma certa distância da camada de névoa. Dessa forma, há um atraso ("Tempo de Propagação Borda-Névoa") de cada andar até a camada de névoa. Os componentes de tempo de propagação não possuem um serviço específico; ou seja, é apenas um componente que causa um atraso na propagação de uma solicitação, emulando um atraso da rede.

Na camada de névoa existe um gateway que é utilizado como ponto de entrada. Ao chegar no gateway, as mensagens podem ser distribuídas seguindo uma estratégia de balanceamento de carga específica. Neste trabalho na seção de análises numéricas nós consideramos a estratégia de distribuição igualitária. Essa distribuição de mensagens não é demorada, desse modo, desconsideramos o tempo para realizá-la. Pela notação Kendall, a rede segue o padrão D/M/c/K/FCFS. A taxa de geração segue um padrão determinístico $(D)$, pois os sensores são calibrados para um intervalo de geração fixo. Os tempos do servidor seguem uma distribuição exponencial. As estações de serviço têm um número (c) de servidores. As respectivas filas têm um tamanho fixo $(K)$ e uma política de ordem de chegada (FCFS). A névoa possui ainda uma estação sink ("Cliente da Névoa") correspondente ao local onde os policiais e bombeiros podem acessar dados sensíveis e em tempo real.

\section{Resultados das Análises}

Esta seção apresenta as análises numéricas realizadas no modelo de filas proposto. A Tabela 3 apresenta a configuração usada para os experimentos. A tag $\mathbf{X}$ indica que o componente não possui definição de capacidade para filas. A coluna de tempo representa o tempo de serviço para os componentes da fila. O tempo de propagação dos componentes representa o tempo para a comunicação entre uma camada e outra. No cenário avaliado foi variado o número de nós na névoa: $2,5,10$ e 15 nós, enquanto a quantidade de nós da borda se manteve fixa (6 nós). Cada nó da borda possui 1 núcleo de processamento, já os nós da névoa possuem 2 núcleos.

A Figura 3 apresenta os resultados considerando diferentes números de nós na névoa. A Figura 3(a) apresenta a taxa de utilização da borda. Como esperado, à medida em que a taxa de chegada aumenta, a taxa de utilização da borda também cresce. Além disso, alterar a quantidade de nós na névoa não influencia no resultado, isso porque a borda não depende de processamento em nós da névoa para realizar suas tarefas. Nesta seção, daremos um exemplo hipotético de uso prático dos resultados para cada métrica. Independente da preferência sobre a utilização da borda, o designer do sistema pode op- 
Tabela 3. Parâmetros Inseridos no Modelo

\begin{tabular}{llll}
\hline Tipo do componente & Componente & Tempo (ms) & Tamanho da Fila \\
\hline Estação com Fila & Dispositivos da Borda & 5 & 50 \\
& Nós da névoa & 30 & 50 \\
\hline \multirow{2}{*}{ Tempo de Propagação } & Borda-névoa 1 & 6 & $\mathrm{X}$ \\
& Borda-névoa 2 & 12 & $\mathrm{X}$ \\
\hline
\end{tabular}

tar por qualquer quantidade de nós na névoa, já que isso não interfere na utilização dos recursos da borda.

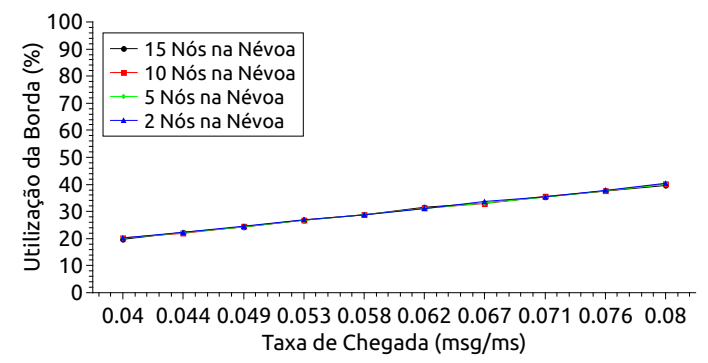

(a) Utilização da borda

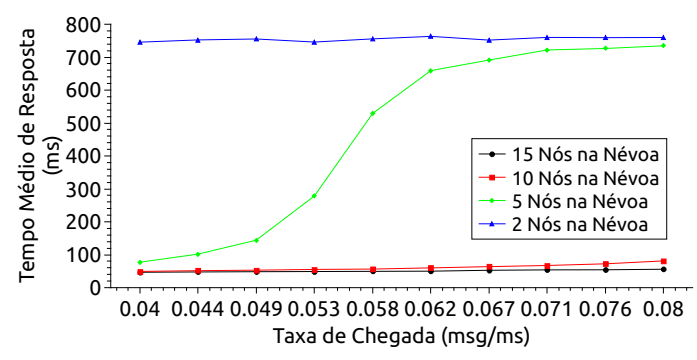

(c) Tempo médio de resposta

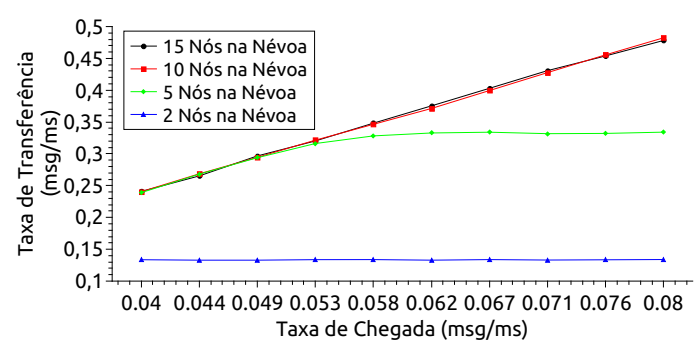

(e) Taxa de transferência

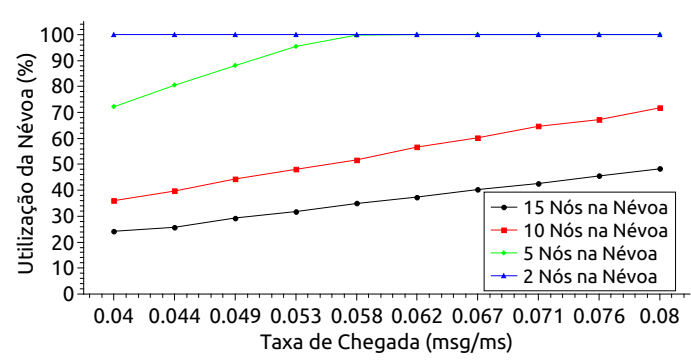

(b) Utilização da névoa

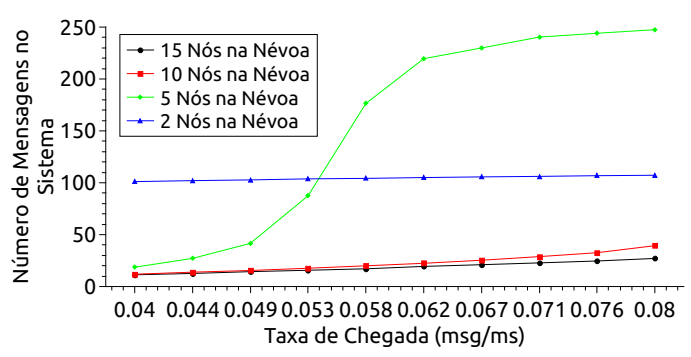

(d) Número de mensagens no sistema

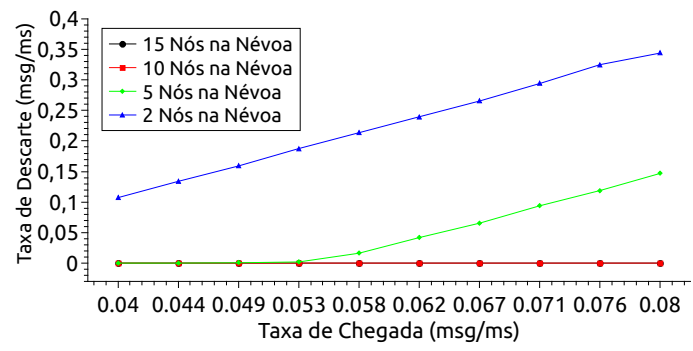

(f) Taxa de descarte

Figura 3. Resultados das análises do modelo considerando diferentes números de nós de névoa.

A Figura 3(b) mostra a taxa de utilização da névoa. Com apenas 2 nós a utilização é de $100 \%$ para todas as taxas de chegada analisadas. Com 5 nós a utilização chega a $100 \%$ com AR igual ou superior a $0,058 \mathrm{msg} / \mathrm{ms}$. A partir de 10 nós a utilização de recursos cai de forma significante e em nenhuma das taxas de chegada observadas os recursos são completamente utilizados. Com 10 nós a utilização máxima é entre $70 \%$ e $80 \%$, e com 15 nós está entre $40 \%$ e 50\%. Imagine que o designer do sistema deseja que a 
utilização da névoa seja maior que $50 \%$, com taxa de chegada de $0,58 \mathrm{msg} / \mathrm{ms}$ e sem taxa de descarte. Neste caso, o ideal seria a configuração com 10 nós na névoa.

A Figura 3(c) apresenta o MRT de todo o sistema. Espera-se que quanto maior o número de nós na névoa, menor seja o MRT, já que o sistema efetua um balanceamento de carga. Esse comportamento pode ser percebido para as configurações observadas nessa análise, no entanto, com 10 ou mais nós na névoa a diferença no MRT pode não ser significante. A configuração com maior MRT é quando a névoa possui 2 nós, pois para todas as taxas de chegadas analisadas o MRT está entre $700 \mathrm{~ms}$ e $800 \mathrm{~ms}$, o que acontece porque a utilização da névoa com essa quantidade de máquinas é de $100 \%$ (ver Figura 3(b)). Com 5 nós o MRT para $A R=0,04 \mathrm{msg} / \mathrm{ms}$ é menor que $100 \mathrm{~ms}$, mas entre $0,049 \mathrm{msg} / \mathrm{ms}$ e $0,071 \mathrm{msg} / \mathrm{ms}$ ocorre um aumento considerável, chegando a $700 \mathrm{~ms}$. Com 10 ou 15 nós na névoa o MRT se mantém relativamente baixo se comparado com as demais configurações, mesmo com $A R=0,08 \mathrm{msg} / \mathrm{ms}$. Imagine que o sistema tenha a taxa de chegada entre $0,04 \mathrm{msg} / \mathrm{ms}$ e $0,049 \mathrm{msg} / \mathrm{ms}$ e o projetista requer que o MRT esteja abaixo de $200 \mathrm{~ms}$. Neste caso, 5 ou mais nós na névoa atendem os requisitos.

A Figura 3(d) exibe o número de mensagens em todo o sistema.É importante observar que as linhas do gráfico possuem curvas similares as do gráfico do MRT (ver Figura 3(c)). Essa semelhança ocorre porque a quantidade de mensagens no sistema está relacionada ao MRT, por exemplo, quando o MRT aumenta é provável que existam mais mensagens no sistema, já que as filas ficam maiores. No entanto com 2 nós na névoa o MRT é maior que na configuração com 5 nós, e ainda assim possui menor número de mensagens no sistema a partir de $A R=0,58 \mathrm{msg} / \mathrm{ms}$. Isso acontece porque com 2 nós o sistema possui taxa de descarte superior as demais configurações, já que possui menor capacidade de processamento e menor quantidade de filas (ver Figura 3(f)).

A Figura 3(e) mostra a taxa de transferência do sistema. É esperado que à medida em que aumenta a taxa de chegada, aumente também a taxa de transferência, já que há mais mensagens chegando. Podemos ver esse comportamento nas configurações com 5,10 e 15 nós, no entanto com 5 nós e $A R=0,58 \mathrm{msg} / \mathrm{ms}$ a taxa começa a estabilizar. A configuração com 2 nós possui taxa de transferência muito inferior às demais, e se manteve estável para todas as taxas de chegada. A estabilidade acontece porque o sistema fica sobrecarregado e começa a descartar mensagens.

A Figura 3(f) apresenta a taxa de descarte de mensagens. Para configurações com 10 ou mais nós, o sistema não descarta nenhuma mensagem, mesmo com $\mathrm{AR}=0,08 \mathrm{msg} / \mathrm{ms}$. Com 5 nós não há descarte de mensagens até $\mathrm{AR}=0,053 \mathrm{msg} / \mathrm{ms}$, mas com taxas de chegada superiores pode descartar mais de $0,1 \mathrm{msg} / \mathrm{ms}$. Com 2 nós o sistema possui taxa de descarte pelo menos $0,1 \mathrm{msg} / \mathrm{ms}$ maior que qualquer outra configuração.

Analisando os resultados dessa seção de forma mais ampla, percebe-se que a utilização da borda da rede não sofre impacto com a alteração da quantidade de nós na névoa, pois não depende do processamento da névoa para funcionar. Para a métrica de tempo médio de resposta, o sistema se comporta de forma estável em todas as configuração da névoa, exceto com 5 nós. Com essa configuração, a partir de $\mathrm{AR}=0,053 \mathrm{msg} / \mathrm{ms}$ o MRT tem aumentos consideráveis, isso acontece porque a utilização de recursos da névoa também cresce, ficando próxima a 100\%. O aumento no MRT faz com que aumente também o número de mensagens no sistema. Nota-se que o segui- 
mento das linhas dos gráficos das duas métricas são semelhantes, mas com AR a partir de $0,058 \mathrm{msg} / \mathrm{ms}$ a configuração com 5 nós passa a ter mais mensagens no sistema que a configuração com 2 nós. Isso pode ser explicado observando os resultados para taxa de transferência e taxa de descarte. Com 2 nós o sistema possui a menor taxa de transferência e a menor capacidade de processamento, o que significa também um menor número de filas, causando aumento na taxa de descarte.

\section{Conclusão}

Este trabalho propôs um modelo de filas do tipo D/M/c/K/FCFS para avaliar o desempenho de infraestruturas de edifícios inteligentes. A arquitetura avaliada inclui sensores IoT e componentes de borda e névoa. O modelo permite a análise de várias métricas, tais como: nível de utilização, taxa de descarte, tempo médio de resposta e taxa de transferência. Além disso, o modelo possui características que não são encontradas em trabalhos relacionados tais como: análise da capacidade de recursos, sensores agrupados por localização e número de núcleos por máquina. Nas análises numéricas foi explorado um cenário onde a capacidade dos recursos da névoa foi variada. Os resultados da análise mostram que a taxa de chegada e a quantidade de recursos disponíveis pode ser bastante influente no desempenho do sistema. O trabalho pode ser útil para que projetistas de sistema neste contexto definam melhor as configurações do sistema de monitoramento de edifícios. Como trabalho futuro, pretende-se estender o modelo incluindo a camada de nuvem e seus componentes, avaliando novos cenários. Além disso, pretendemos desenvolver e implantar um sistema real para comparar seu desempenho com os resultados obtidos com o modelo.

\section{Referências}

Ajao, L. A., Agajo, J., Umar, B. U., Agboade, T. T., and Adegboye, M. A. (2020). Modeling and implementation of smart home and self-control window using fpga and petri net. In 2020 IEEE PES/IAS PowerAfrica, pages 1-5. IEEE.

Arbib, C., Arcelli, D., Dugdale, J., Moghaddam, M., and Muccini, H. (2019). Real-time emergency response through performant iot architectures. In International Conference on Information Systems for Crisis Response and Management (ISCRAM).

Attia, M. B., Nguyen, K.-K., and Cheriet, M. (2019). Dynamic qoe/qos-aware queuing for heterogeneous traffic in smart home. IEEE Access, 7:58990-59001.

Fanti, M. P., Mangini, A. M., and Roccotelli, M. (2014a). A petri net model for a building energy management system based on a demand response approach. In 22nd Mediterranean Conference on Control and Automation, pages 816-821.

Fanti, M. P., Mangini, A. M., and Roccotelli, M. (2018). A simulation and control model for building energy management. Control Engineering Practice, 72:192-205.

Fanti, M. P., Mangini, A. M., Ukovic, W., Lesage, J., and Viard, K. (2014b). A petri net model of an integrated system for the health care at home management. In 2014 IEEE International Conference on Automation Science and Engineering (CASE), pages 582587.

Fishman, G. S. (2013). Discrete-event simulation: modeling, programming, and analysis. Springer Science \& Business Media. 
Garcia, M., Konios, A., and Nugent, C. (2018). Modelling activities of daily living with petri nets. In 2018 IEEE International Conference on Pervasive Computing and Communications Workshops (PerCom Workshops), pages 866-871.

Jafarnejad Ghomi, E., Rahmani, A. M., and Qader, N. N. (2019). Applying queue theory for modeling of cloud computing: A systematic review. Concurrency and Computation: Practice and Experience, 31(17):e5186.

Memon, R. A., Li, J. P., and Ahmed, J. (2019). Simulation model for blockchain systems using queuing theory. Electronics, 8(2):234.

Mena, D. M., Papapanagiotou, I., and Yang, B. (2018). Internet of things: Survey on security. Information Security Journal: A Global Perspective, 27(3):162-182.

Nabih, A. K., Gomaa, M. M., Osman, H. S., and Aly, G. M. (2011). Modeling, simulation, and control of smart homes using petri nets. International Journal of Smart Home, $5(3): 1-14$.

Novák, M., Biňas, M., and Jakab, F. (2012). Unobtrusive anomaly detection in presence of elderly in a smart-home environment. In 2012 ELEKTRO, pages 341-344.

Oueida, S., Kotb, Y., Aloqaily, M., Jararweh, Y., and Baker, T. (2018). An edge computing based smart healthcare framework for resource management. Sensors, 18(12):4307.

Said, O. and Masud, M. (2013). Towards internet of things: Survey and future vision. International Journal of Computer Networks, 5(1):1-17.

Sokullu, R., Akkaş, M. A., and Demir, E. (2020). Iot supported smart home for the elderly. Internet of Things, 11:100239.

Wang, B. C., Sechilariu, M., and Locment, F. (2013). Power flow petri net modelling for building integrated multi-source power system with smart grid interaction. Mathematics and Computers in Simulation, 91:119-133.

Wu, T., Zhou, P., Liu, K., Yuan, Y., Wang, X., Huang, H., and Wu, D. O. (2020). Multiagent deep reinforcement learning for urban traffic light control in vehicular networks. IEEE Transactions on Vehicular Technology, 69(8):8243-8256.

Xu, H., He, Y., Sun, X., He, J., and Xu, Q. (2020). Prediction of thermal energy inside smart homes using iot and classifier ensemble techniques. Computer Communications, 151:581-589. 